Louisiana State University

LSU Digital Commons

Faculty Publications

Department of Chemistry

10-13-2020

\title{
Dynamics of PDMS- g-PDMS Bottlebrush Polymers by Broadband Dielectric Spectroscopy
}

\author{
Bruno Jakobi \\ Louisiana State University \\ Karin J. Bichler \\ Louisiana State University \\ Anna Sokolova \\ Australian Nuclear Science and Technology Organisation \\ Gerald J. Schneider \\ Louisiana State University
}

Follow this and additional works at: https://digitalcommons.Isu.edu/chemistry_pubs

\section{Recommended Citation}

Jakobi, B., Bichler, K., Sokolova, A., \& Schneider, G. (2020). Dynamics of PDMS- g-PDMS Bottlebrush

Polymers by Broadband Dielectric Spectroscopy. Macromolecules, 53 (19), 8450-8458. https://doi.org/

10.1021/acs.macromol.0c01277

This Article is brought to you for free and open access by the Department of Chemistry at LSU Digital Commons. It has been accepted for inclusion in Faculty Publications by an authorized administrator of LSU Digital Commons.

For more information, please contact ir@lsu.edu. 


\section{Dynamics of PDMS-g-PDMS Bottlebrush Polymers by Broadband}

$$
\text { Bruno Jakobi }{ }^{*} \text {, Karin J. Bichler }{ }^{2}, \text { Anna Sokolova }^{3} \text {, Gerald J. Schneider }{ }^{1,2^{*}}
$$

$4 \quad{ }^{1}$ Louisiana State University, Department of Chemistry, 70803 Baton Rouge, USA

$5 \quad 2$ Louisiana State University, Department of Physics and Astronomy, 70803 Baton Rouge, USA

$6 \quad{ }^{3}$ Australia Center for Neutron Scattering, ANSTO, New Illawarra Road, Lucas Heights 2234, Aus-

7 tralia

\section{Email:}

11 Bruno Jakobi: bjakob1@1su.edu

12 Gerald J. Schneider: gjschneider@1su.edu

\section{Table of Content Figure}
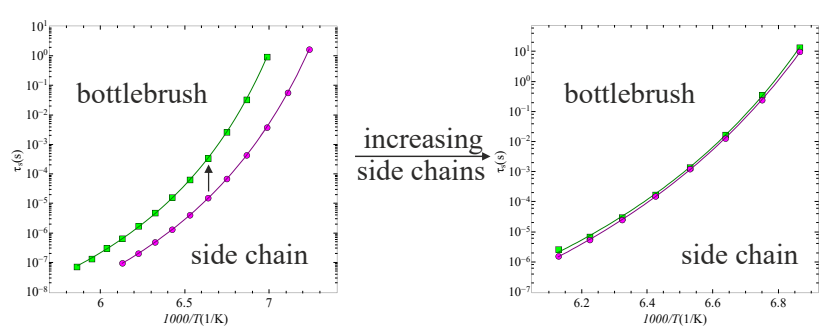

17 Keywords

18 Bottlebrush, Polymer, PDMS, Segmental Dynamics, Dielectric Spectroscopy, Neutron Scattering 


\section{Abstract}

Poly(dimethylsiloxane) (PDMS) based bottlebrush polymers, PDMS-g-PDMS, have been synthesized by anionic polymerization in combination with a condensation based grafting reaction. Bottlebrush polymers show intriguing features, e.g., extremely low viscosities. Hereby studies of their dynamics are rare. Therefore, we focus on the segmental relaxation by broadband dielectric spectroscopy. An increasing cross-sectional radius proportional to the increasing side chain length has been observed by small-angle neutron scattering over three samples. A comparison of the segmental relaxation times of the bottlebrushes with the respective linear chains reveals slower dynamics in the former. For longer chains this effect vanishes.

\section{Introduction}

Bottlebrush polymers count to the branched polymers. Hereby, a high number of linear side chains are covalently bonded to a linear backbone, preventing the backbone from adopting a random coil conformation. ${ }^{1}$ Changing the ratio of side chain to backbone length strongly influences the shape of bottlebrush polymers. This enables the synthesis of various structures ranging from elongated to spherical objects. ${ }^{2-4}$ Several possible applications originate from the structural features, ranging from lubricants, to solvent free elastomers ${ }^{5}$ up to surface modifications ${ }^{6}$ or drug de- $^{-}$ livery agents ${ }^{7}$.

Investigations based on the dynamics are rare. So far, the main focus of study is the difference in flow behavior compared to linear polymers, as determined by rheology.$^{8-9}$ Viscosity of polymers is related to their glass transition temperature, $T_{g}$, defined by $T_{g}=T\left(\eta_{0}=10^{12} \mathrm{~Pa} \mathrm{~s}\right) \cdot{ }^{10} \mathrm{~A}$ similar expression exists for the segmental relaxation time, $\tau_{s}$, often measured by dielectric spectroscopy, i.e., $T_{g}=T\left(\tau_{s}=100 \mathrm{~s}\right) .{ }^{11}$ However, studies based on the segmental relaxation behavior of 
43 homopolymer bottlebrushes compared to their linear side chains are missing. The dielectric relax-

44 ation properties of heteropolymer bottlebrushes have been investigated in comparison to their re-

45 spective backbone and side chain polymers. ${ }^{12-14}$

46 In this publication we are focusing on the segmental relaxation of poly(dimethylsiloxane) based

47 bottlebrush, PDMS-g-PDMS, polymers compared to their respective linear PDMS side chains. For

48 that, a synthesis route towards PDMS-g-PDMS bottlebrush polymers by the grafting-to method has

49 been developed. This approach enables us to investigate the relaxation behavior of the single com-

50 ponents prior to the grafting process. Furthermore, by using the same polymer for backbone and

51 side chains, we minimize the risk of nano- or microphase separation. These phenomena are known

52 to influence or constrain the dynamics of polymers with side chains, e.g., poly(alkylene oxide)s

53 and poly(alkyl acrylate)s. ${ }^{15-16}$ 


\section{Analysis}

\section{Small-Angle Neutron Scattering}

58 For systems having a Kuhn length, $\ell_{k}$, and contour length, $L$, much larger than the cross-sec-

59 tional radius, $R$, the formfactor can be obtained by the decoupling approximation. Hereby, the

60 backbone contour length (Figure 1 left), is treated separately from the side chains, i.e., the cross

61 section (Figure 1 right). ${ }^{17}$

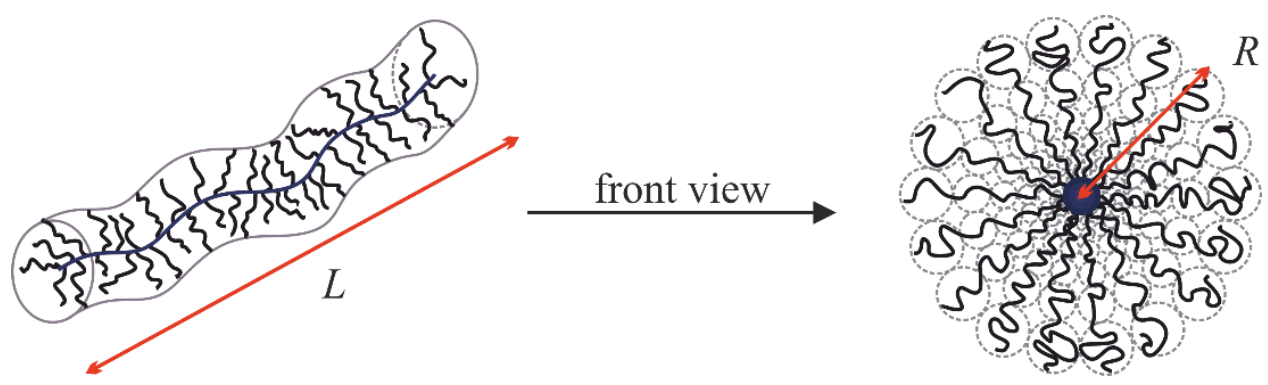

Figure 1. Pictorial representation of an elongated bottlebrush polymer, showing the side (left) and front view (right), with contour length, $\mathrm{L}$, and cross-sectional radius, $\mathrm{R}$.

The backbone is assumed to have an expanded conformation due to the high grafting density.

63 However, because of the attached side chains, the overall structure is still flexible. Therefore, the

64 backbone can be approximated by a semiflexible polymer chain, whereby the side chains are con-

65 sidered via the cross section by applying the Fourier transformation of the star like density profile,

66 including the Flory exponent $v^{4,18}$

$$
\varphi(r)_{\text {star }}=\frac{r^{\frac{1-3 v}{v}}}{\left(1+\exp \left(\frac{r-R}{\sigma R}\right)\right)}
$$

67 Here, the radial monomer density distribution ${ }^{19}$ is multiplied by the Fermi cut-off function to ensure

68 a smooth decay at the outermost radius $R$ including the smearing parameter $\sigma$.

The total scattering function for elongated bottlebrush polymer can be written as ${ }^{4}$ 


$$
I(Q)=\Phi V_{\text {total }} \Delta \rho^{2} \cdot\left(P_{S P C}(Q) \cdot P_{C S}(Q)+a \cdot \frac{P_{b l o b}(Q)}{1+v_{E V} P_{b l o b}(Q)}\right)
$$

70 with volume concentration, $\Phi$, the contrast, $\Delta \rho$, and the total volume, $V_{\text {total }}$, of the polymer, in-

71 cluding all side chains and the backbone. It can be determined by using the molecular weight, $M_{w}$,

$$
V_{\text {total }}=V_{\text {side chain }} \cdot f+V_{\text {backbone }}
$$

72 with number of side chains, $f$, and volume, $V_{i}=\frac{M_{w_{i}}}{\varrho_{i} N_{A}}$, associated with side chains and backbone

73 and the respective mass density, $\varrho_{i}$. The form factor $P_{S P C}(Q)$ stands for an infinitely thin, semi-

74 flexible polymer chain including excluded volume effects as described by Pedersen et al. ${ }^{20}$,

75 method 3, and Chen et al. ${ }^{18}$ The final model for the semiflexible polymer chain has three adjustable

76 parameters, the Kuhn length, $\ell_{k}$, the Flory exponent, $v$, and the cylinder contour length, $L$.

77 The form factor of the cross section, $P_{C S}(Q)$, can be calculated by a two-dimensional Fourier

78 transformation of the star like density profile, equation (1). This reads as

$$
P_{C S}(Q)=\left|\frac{\int_{0}^{R} \varphi(r)_{\text {star }} \cdot J_{0}(Q r) r d r}{\int_{0}^{R} \varphi(r)_{\text {star }} r d r}\right|^{2}
$$

with $J_{0}$ denoting the zeroth-order Bessel function of first kind and $r$ the radial distance from the

80 backbone. The dimension of our bottlebrush cross section is finite, therefore the outer radius, $R$,

81 can be taken for the upper integration limit of the Fourier transformation instead of infinity. For the

82 description of the internal structure within the cross section, a blob contribution, scaled with the

83 prefactor $a$, is added to the form factor, considering the excluded volume, $v_{E V}$. With $v_{E V}$ overlap-

84 ping chains are considered and subtracted from the overall contribution. The form factor for the 85 blob contribution, $P(Q)_{b l o b}$, can be described as: 


$$
P(Q)_{b l o b}=\exp \left(-\frac{Q^{2} \xi^{2}}{3}\right)+\left(\frac{d_{f}}{\xi^{d_{f}}}\right) \Gamma\left(\frac{d_{f}}{2}\right)\left(\frac{\operatorname{erf}\left(\frac{Q k \xi}{\sqrt{6}}\right)^{3}}{Q}\right)^{d_{f}}
$$

86 Here, $k=1.06$ is an empirical constant, $\xi$ the radius of gyration and $d_{f}$ the fractal dimension of

87 the scattered particle which is typically $d_{f}=2$ for melt or $\Theta$-conditions or $d_{f}=1.7$ for so called

88 good solvent conditions. This parameter is connected to the Flory exponent, $v$, with $d_{f}=1 / v$. The

89 typical value for melt or $\Theta$-condition is $v=0.5$, whereby for good solvent $v=0.588$, as predicted

90 by perturbation theory. ${ }^{21}$

\section{Broadband Dielectric Spectroscopy}

The PDMS based bottlebrush polymers have only a dipole moment perpendicular to the polymer chain, in both, backbone and the side chains, and are thus type B polymers. Therefore, by using

95 dielectric spectroscopy, only the segmental dynamics are probed.

97 function, $\epsilon^{*}(\omega)$, is defined as

$$
\epsilon^{*}(\omega)=\epsilon_{\infty}+\frac{\Delta \epsilon}{1+i \omega \tau_{D}}
$$

98 with $\tau_{D}$ as the characteristic relaxation time of the system. ${ }^{22}$ However, most of the time, the ob-

99 served processes are non-ideal and the Debye model is not applicable. The empirical Havriliak-

100 Negami function (HN), an adaption of the Debye model for non-ideal, i.e., asymmetric, relaxation 101 processes, is used to describe the dielectric data in the frequency domain. ${ }^{23}$

$$
\epsilon_{H N}^{*}(\omega)=\epsilon_{\infty}+\frac{\Delta \epsilon}{\left(1+\left(i \omega \tau_{H N}\right)^{\beta}\right)^{\gamma}}
$$


102 Here, $\beta$ and $\gamma$ are shape parameters, describing the asymmetric broadening of the relaxation

103 peak with the restrictions of $0<\beta \leq 1$ and $0<\gamma \cdot \beta \leq 1$. This complex function can also be split

104 in a real and an imaginary part

$$
\begin{aligned}
& \epsilon_{H N}^{\prime}(\omega)=\epsilon_{\infty}+\Delta \epsilon r(\omega) \cos [\gamma \psi(\omega)] \\
& \epsilon_{H N}^{\prime \prime}(\omega)=\Delta \epsilon r(\omega) \sin [\gamma \psi(\omega)]
\end{aligned}
$$

105 with

$$
\begin{aligned}
& r(\omega)=\left[1+2\left(\omega \tau_{H N}\right)^{\beta} \cos \left(\frac{\beta \pi}{2}\right)+\left(\omega \tau_{H N}\right)^{2 \beta}\right]^{-\frac{\gamma}{2}} \\
& \psi(\omega)=\arctan \left[\frac{\sin \left(\frac{\beta \pi}{2}\right)}{\left(\omega \tau_{H N}\right)^{-\beta}+\cos \left(\frac{\beta \pi}{2}\right)}\right]
\end{aligned}
$$

106 This results in the Havriliak-Negami relaxation time $\tau_{H N}{ }^{22}$ However, $\tau_{H N}$ does not represent

107 the "real" relaxation time $\tau_{s}$, because it is slightly shifted due to the asymmetry of the relaxation

108 peak. In order to correct this, the following equation needs to be applied. ${ }^{24-27}$

$$
\frac{1}{\tau_{s}}=\frac{1}{\tau_{H N}}\left[\sin \left(\frac{\beta \pi}{2+2 \gamma}\right)\right]^{\frac{1}{\beta}}\left[\sin \left(\frac{\beta \gamma \pi}{2+2 \gamma}\right)\right]^{-\frac{1}{\beta}}
$$

109 The temperature dependence of the segmental relaxation can be described with the

110 Vogel-Fulcher-Tammann (VFT) equation.

$$
\tau_{s}=\tau_{\infty} \exp \left(\frac{A}{T-T_{0}}\right)
$$




\section{Experimental Methods}

114 Gel-Permeation Chromatography with Multi Angle Laser Light Scattering (GPC-MALLS)

115 For all Gel-Permeation Chromatography with Multi-Angle Laser Light Scattering (GPC-

116 MALLS) measurements a GPC with two $500 \mathrm{~mL}$ HL syringe pumps from Teledyne Isco Inc., a

117 Rheodyne manual injection valve from Kinesis Inc., three Phenogel $5 \mu \mathrm{m}$ columns $(300 \times$

$1187.8 \mathrm{~mm}$ ) from Phenomenex, a Dawn Eos 18-angle static light scattering detector from Wyatt Tech-

119 nology Corporation, and a L-7490 differential Refractive Index (dRI) detector from Hitachi were

120 used. The Astra software package was used for data collection and treatment.

\section{Small-Angle Neutron Scattering (SANS)}

Small-angle neutron scattering (SANS) experiments have been performed with the Bilby in-

124 struments at the Australian Centre for Neutron Scattering (ACNS) in Sydney, Australia. ${ }^{28-29}$ The

125 monochromatic mode has been used, with a wavelength of $\lambda=6 \AA$ and a resolution of $\frac{\Delta \lambda}{\lambda}=10 \%$.

126 Bilby has two detector carriages which can move independently within the vacuum vessel. The

127 detector carriages consist of one single rear detector and a complex front detector assembled of

128 four equally sized panels, called curtains. Hereby, distances from the sample to the rear detector,

129 the horizontal and the vertical curtains of $13.5 \mathrm{~m}, 1.9 \mathrm{~m}$, and $0.9 \mathrm{~m}$ were used, with a separation

130 of the left and right curtain of $0.175 \mathrm{~m}$ and $0.350 \mathrm{~m}$ and those of the top and bottom curtain equals

131 to $0.150 \mathrm{~m}$ and $0.030 \mathrm{~m}$. This allows to capture the $Q$-range from $0.003 \AA^{-1}$ to $0.56 \AA^{-1}$ with the

132 momentum transfer $Q=\frac{4 \pi}{\lambda} \sin \left(\frac{\vartheta}{2}\right)$ and scattering angle $\vartheta$, within one measurement, without

133 changing the detector arrangement. The data reduction was performed using Bilby specific suitable

134 routines in MANTID. ${ }^{30}$ After correction for empty cell scattering, transmission, and detector re-

135 sponse, the data sets were radially averaged and transformed onto an absolute scale. All samples 
136 were additionally background corrected by subtracting the corresponding contributing volume frac-

137 tion of the solvent.

\section{Broadband Dielectric Spectroscopy}

140 The dielectric spectroscopy measurements were performed with a Broadband Dielectric Alpha

141 Analyzer from Novocontrol $\mathrm{GmbH}$. The instrument operates in a frequency range of

$14210^{-2} \mathrm{~Hz}-10^{6} \mathrm{~Hz}$. The available temperature range of $T=-150{ }^{\circ} \mathrm{C}$ to $T=+500{ }^{\circ} \mathrm{C}$ is con-

143 trolled by a Quatro Cryosystem with a manufacturer specified accuracy of $0.1^{\circ} \mathrm{C}$ by using an evap-

144 orated nitrogen stream. For the measurements, a temperature range of $T=-140{ }^{\circ} \mathrm{C}$ to

$145 T=-90{ }^{\circ} \mathrm{C}$ with an increment of $\Delta T=2.5^{\circ} \mathrm{C}$ was enough to move the segmental dynamics

146 through the available frequency range. A waiting time of three minutes was set prior to each spec-

147 trum measurement, ensuring a temperature stability equal or better than $0.1^{\circ} \mathrm{C}$. To avoid crystalli-

148 zation, the sample was rapidly cooled down to $T=-140{ }^{\circ} \mathrm{C}$ and measurements at all temperatures

149 were conducted in the order from low to high temperatures. 


\section{Results and Discussion}

\section{Synthesis}

163 In the synthesis of PDMS based homopolymer bottlebrush the focus has mainly been on the 164 grafting of living PDMS, with active lithium silanolate chain ends, onto a chlorosilane functional165 ized backbone, i.e., poly(methyl-2-chlorodimethylsilylethylsiloxane) or poly(methylchlorosilox166 ane) (PMClS). ${ }^{31-32}$ These reactions suffer from side reactions, typically suppressed in the anionic

167 ring opening polymerization of PDMS. In this paper we explored a mild grafting reaction based on 168 the iterative growth of linear oligo(dimethlsiloxane)s in combination with the palladium catalyzed 169 chlorination of PMHS as a viable alternative for the use of living PDMS (Scheme 1). ${ }^{33-34}$

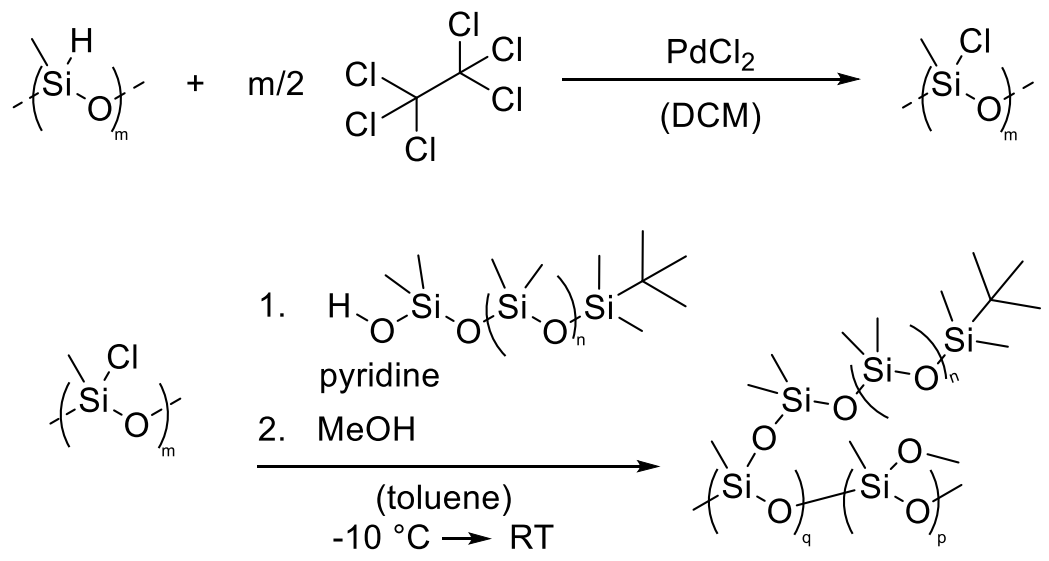

Scheme 1. Palladium catalyzed chlorination (top) and condensation based grafting reaction (bottom).

The precursor polymer, i.e., poly(methylhydrosiloxane) (PMHS), has been synthesized via the

172 known cationic ring opening equilibration of tetramethylcyclotetrasiloxane and was split into frac-

173 tions via precipitative fractionation with toluene as a solvent and acetonitrile as a non-solvent. For 174 the activation of the backbone, a chlorination reaction of silanes catalyzed by palladium chloride 175 with hexachloroethane as a chlorine source was adapted from Pongkittiphan et al. ${ }^{33}$ The proposed 176 mechanism involves the catalyst activation under production of hydrogen chloride. ${ }^{33}$ To prevent 
177 thermodynamic re-equilibration of the siloxane bonds, hydrogen chloride needs to be removed 178 from the reaction. This was achieved by activating a $\mathrm{PdCl}_{2} / \mathrm{hexachloroethane} \mathrm{mixture} \mathrm{with} \mathrm{chloro-}$ 179 dimethylsilane followed by partial drying in vacuum. The reaction in pure dichloromethane re180 sulted in a degree of chlorination of $99.7 \%$ as determined by the disappearance of the $\mathrm{SiH}$ signal 181 in the ${ }^{1} \mathrm{H}$ NMR spectrum. The chlorination was used for the in situ functionalization of PMHS 182 directly before the grafting of side chains, avoiding the need for purification by filtration as needed 183 in the chlorination with trichloroisocyanuric acid. ${ }^{32}$

184 Van Genabeek et al. reported the synthesis of monodisperse, low molecular weight PDMS by 185 the repeated condensation of a oligo(dimethylsiloxane)s with silanol and chlorosilane chain ends, 186 followed a reactivation of the chain ends for further condensation. ${ }^{34}$ The condensation product, 187 hydrochloric acid, is trapped by the addition of pyridine. ${ }^{34}$ This concept is transferred towards the synthesis of bottlebrushes. The chlorosilane functionalized oligomer is replaced with PMClS as the electrophile and the silanol functionalized oligomer by a silanol terminated PDMS.

190 The applicability of the reaction is demonstrated via the synthesis of three bottlebrushes with 191 long, middle, and short side chains, i.e., with 155, 25, and 4 repeating units, respectively (Table 192 1). Hereby, the long and middle side chains possess bulky tert-butyl end groups, whereas the short 193 ones have slim $\mathrm{SiH}$ end groups. The grafting reaction results in similar grafting densities for the 194 middle and long side chains and almost complete grafting for the shortest side chains. The molec195 ular weight of the fraction of unreacted polymer remains unchanged after the grafting reaction, 196 compared to the ungrafted side chains, as seen on the chromatograms of the bottlebrush with the 197 longest side chains in Figure 2. The other chromatograms and ${ }^{1} \mathrm{H}$ NMR spectra can be found in 198 Figure S1-S6 in the Supporting Information. The peak corresponding to the bottlebrush in the crude 199 product mixture (black chromatograms) shows a low molecular weight tail. This part of the product 200 mixture is removed after fractionation. 


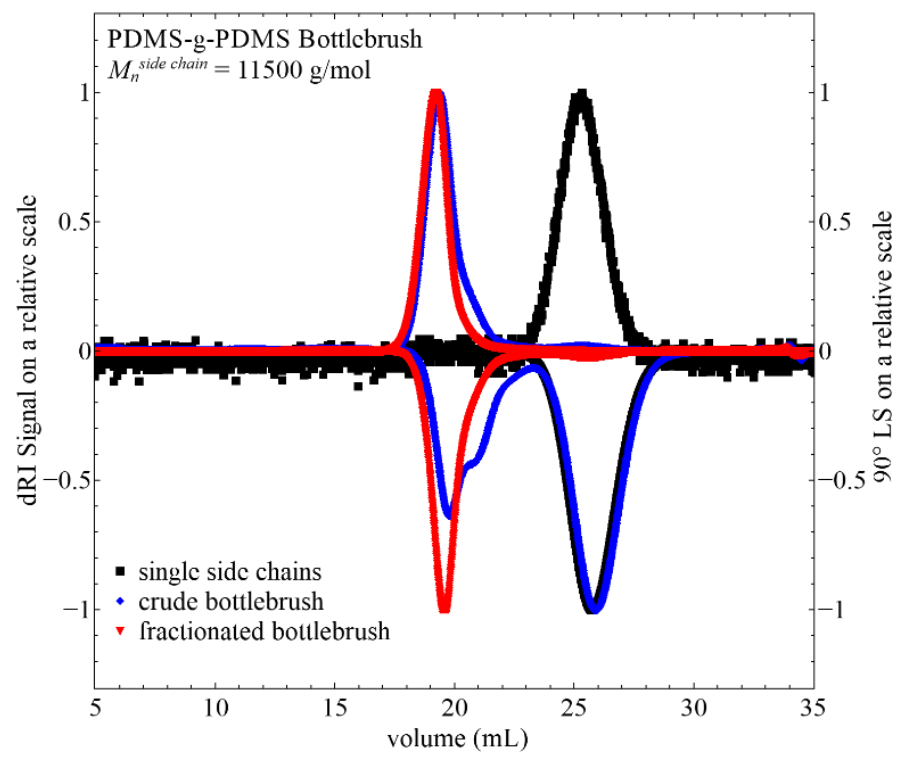

Figure 2. Differential Refractive Index (dRI) (down peak) and $90^{\circ}$ Light Scattering (LS) (up peak) signal of the GPC-MALLS chromatogram on a relative scale for the single side chain (black), crude bottlebrush (blue), and fractionated bottlebrush (red) for the longest side chains.

Table 1. Number average molecular weight, $M_{n}$, PDI, and the grafting density, $\rho^{*}$, of the three bottlebrush samples and their constituents.

\begin{tabular}{c|c|c}
\hline Name & $\begin{array}{c}\text { single chain } \\
M_{n}(\mathrm{~g} / \mathrm{mol})(\mathrm{PDI})\end{array}$ & $\begin{array}{c}\text { bottlebrush } \\
M_{n}(\mathrm{~kg} / \mathrm{mol})(\mathrm{PDI})\left[\rho^{*}\right]\end{array}$ \\
\hline Short & $298(1.0)$ & $95(1.3)[89]$ \\
\hline Middle & $1800(1.2)$ & $157(1.1)[30]$ \\
\hline Long & $11500(1.1)$ & $1106(1.2)[41]$ \\
\hline Backbone-short & $16500(1.1)$ & --- \\
\hline Backbone-middle/long & $13500(1.2)$ & - \\
\hline
\end{tabular}




\section{Small-Angle Neutron Scattering}

206 A low concentration, $\Phi=0.5 \%$, of the bottlebrush polymers in deuterated cyclohexane is

207 measured to identify the pure form factor, $P(Q)$. For the sample, having the shortest side chains,

$208 M_{n}^{\text {side }}$ chain $=298 \mathrm{~g} / \mathrm{mol}$ (blue circles), two kinks appear, as seen in Figure 3 (blue circles). While

209 the first one is located at $Q \sim 0.01 \AA^{-1}$, the second one is at $Q \sim 0.2 \AA^{-1}$. In the region between these

210 two kinks an intensity dependence of $I \propto Q^{-1.7}$ is found. This power law of 1.7 suggests the pres-

211 ence of elongated shapes with a semi-flexible structure in good solvent conditions. ${ }^{4}$ Hereby, the

212 first kink corresponds to the length, whereas the second one is attributed to the cross-sectional

213 radius. With increasing side chain length, the second kink from $Q \sim 0.2 \AA^{-1}$ moves to smaller $Q$

214 (Figure 3, green squares), while the kink at lower $Q$ stays the same. This effect is caused by an

215 increase of the cross-sectional radius through the longer side chains, while the overall length of the

216 polymer stays the same. Both together show the independent modification of length and cross-

217 sectional radius. Increasing the side chain length further to $M_{n}^{\text {side chain }}=11500 \mathrm{~g} / \mathrm{mol}$ shifts both

218 kinks even closer together resulting in a direct transition from low to intermediate $Q$-values, which

219 suggest the appearance of an almost spherical shape (Figure 3, red diamonds).

220 All samples can be well described by the form factor, $P(Q)$, for elongated bottlebrush polymers,

221 equation (2). The resulting fit parameters are summarized in Table 2. 


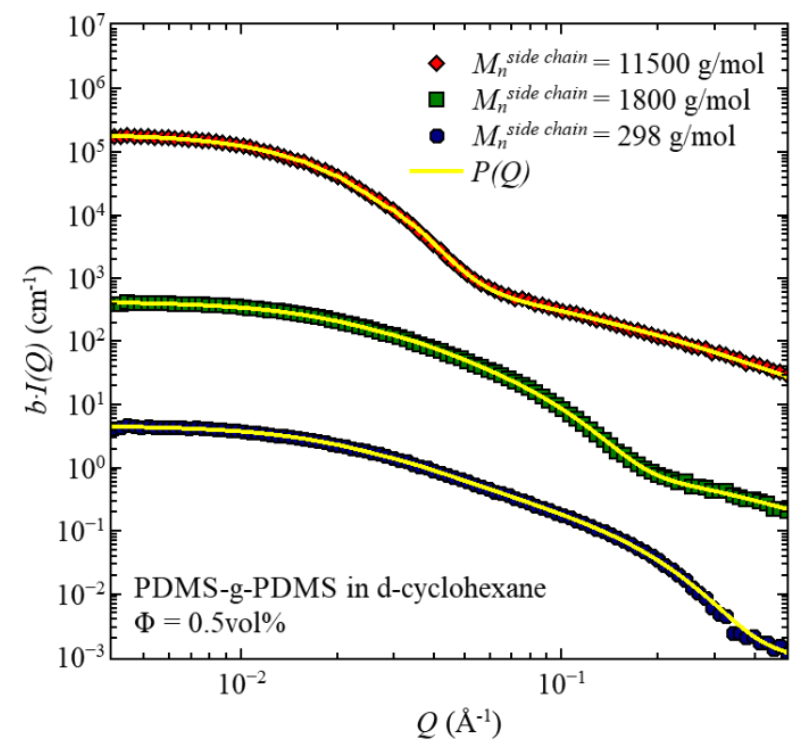

Figure 3. Scattering intensity, $I(Q)$, vs. momentum transfer, $Q$, of the three different PDMS-gPDMS bottlebrush polymers in d-cyclohexane. Solid lines represent the description with the form factor $P(Q)$. For clarity, the data are shifted by a factor $b$ in y-direction. Blue circles $b=1$, green squares $b=50$, and red diamonds $b=5000$. Error bars are within symbol size and omitted.

Table 2. Contour length, $L$, radius, $R$, Kuhn length, $\ell_{k}$, and blob size, $\xi$, of the three bottlebrush polymers. All errors are smaller than $1 \%$. An additional table with the remaining fit parameters can be found in the Supporting Information.

\begin{tabular}{c|c|c|c}
\hline Name & Short & Middle & Long \\
\hline Length $L(\AA)$ & 797.0 & 796.7 & 840.7 \\
\hline Radius $R(\AA)$ & 4.8 & 8.7 & 47.2 \\
\hline Kuhn length $\ell_{k}(\AA)$ & 43.6 & 43.6 & 42.8 \\
\hline Blob size $\xi(\AA)$ & 2.7 & 7.5 & 18.9 \\
\hline
\end{tabular}

The fit parameters illustrate the independence of the Kuhn length, $\ell_{k}$, from the side chain length

225 while the contour length, $L$, increases. This is a consequence of the attachment of the side chains,

226 as those located at the extremities of backbone cause its extension. This effect exists for all three

227 samples but is most evident for the bottlebrush with the longest side chain. Only the radius and the 
blob size are substantially influenced by the side chain variation. The Kuhn length of PDMS-g-

229 PDMS seems to be significantly larger than the one of linear PDMS, $\ell_{k}^{P D S M}=13 \AA .{ }^{35}$ This obser-

230 vation appears to be a common feature shared by a variety of bottlebrush polymers if compared to

231 their respective linear counterparts. ${ }^{4}$

233 Broadband Dielectric Spectroscopy

234 All samples, together with the single side chains, i.e., PDMS-g-PDMS bottlebrush and linear

235 PDMS, have been measured with dielectric spectroscopy. Since PDMS is a so-called type B poly-

236 mer, dielectric spectroscopy has access to the segmental relaxation for the bottlebrush polymers

237 and the single side chains, resulting in information about the segmental relaxation times, $\tau_{s}$. How-

238 ever, the majority of the dipole moments are located in the side chains; therefore, the segmental

239 relaxation of the bottlebrush polymers is dominated by the side chains.

240 This publication sets the focus on the pure segmental relaxation. However, the bottlebrush pol-

241 ymers also show the influence of the cold crystallization on the segmental relaxation, i.e., the $\alpha_{c^{-}}$

242 relaxation, as known for linear PDMS. ${ }^{36}$ This effect intensifies with increasing molecular weight

243 of the side chains. In our samples, the $\alpha_{c}$-relaxation is well pronounced only for the sample with

244 the longest side chains, $M_{n}^{\text {side chain }}=11500 \mathrm{~g} / \mathrm{mol}$ (Supporting Information Figure S8). For the

245 other two samples, the crystallization is marginal due to the low molecular weight and does not

246 allow to be extract a relaxation time. Since we are focusing on the pure segmental relaxation, the

$247 \alpha_{c}$-relaxation will not be discussed further.

248 The typical relaxation spectra obtained by dielectric spectroscopy for the PDMS-g-PDMS bot-

249 tlebrush polymers (top row) and the respective linear PDMS side chains (bottom row) are shown

250 in Figure 4. 

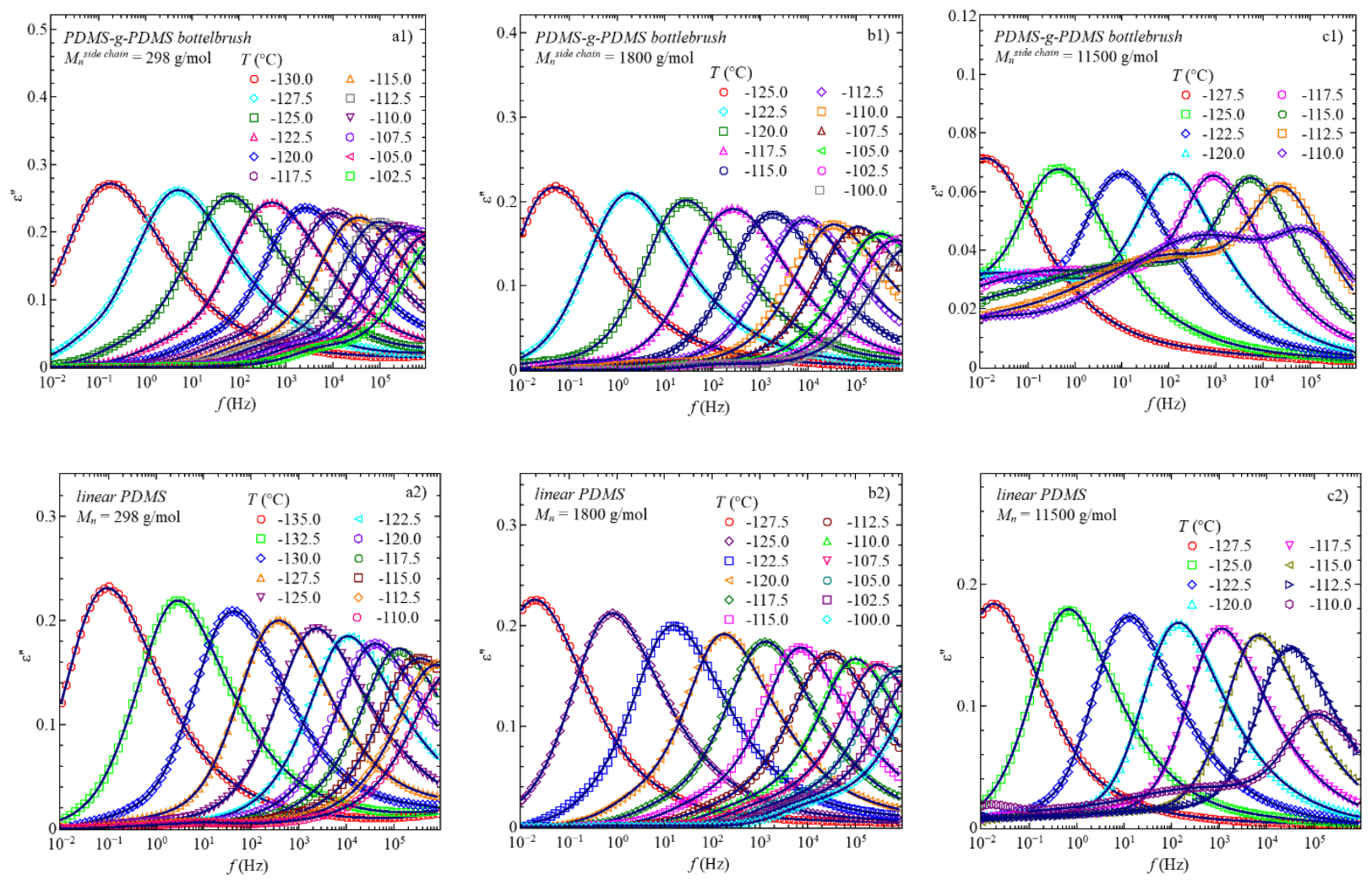

Figure 4. Imaginary part of the permittivity, $\epsilon^{\prime \prime}$, vs. frequency, $f$, for the PDMS-g-PDMS bottlebrush polymers (top) compared with the respective linear PDMS side chains (bottom). Top row: $\quad$ a1: $\quad M_{n}^{\text {side chain }}=298 \mathrm{~g} / \mathrm{mol}, \quad$ b1: $\quad M_{n}^{\text {side chain }}=1800 \mathrm{~g} / \mathrm{mol}$, c1: $M_{n}^{\text {side chain }}=11500 \mathrm{~g} / \mathrm{mol}$. Bottom row: $\mathrm{a} 2: M_{n}=298 \mathrm{~g} / \mathrm{mol}, \mathrm{b} 2: M_{n}=1800 \mathrm{~g} / \mathrm{mol}$, c2: $M_{n}=11500 \mathrm{~g} / \mathrm{mol}$. Solid lines represent the best descriptions with Havriliak-Negami functions.

Here, in bottlebrush polymers as well as in the single side chains, a well pronounced relaxation

254 peak is visible which shifts to higher frequencies with increasing temperature. This is associated

255 with a decrease in relaxation time, $\tau_{s}$, which is the reciprocal value of the frequency at the peak

256 maximum. The data can be well described with a combination of Havriliak-Negami functions.

257 Normalizing the dielectric permittivity as well as the frequency to one allows to analyze the

258 influence of the temperature on the shape of the relaxation peak (Figure 5). This comparison shows

259 no effect of temperature on the segmental relaxation other than a changing relaxation time. This

260 applies to all samples, regardless of the architecture of the polymer. 

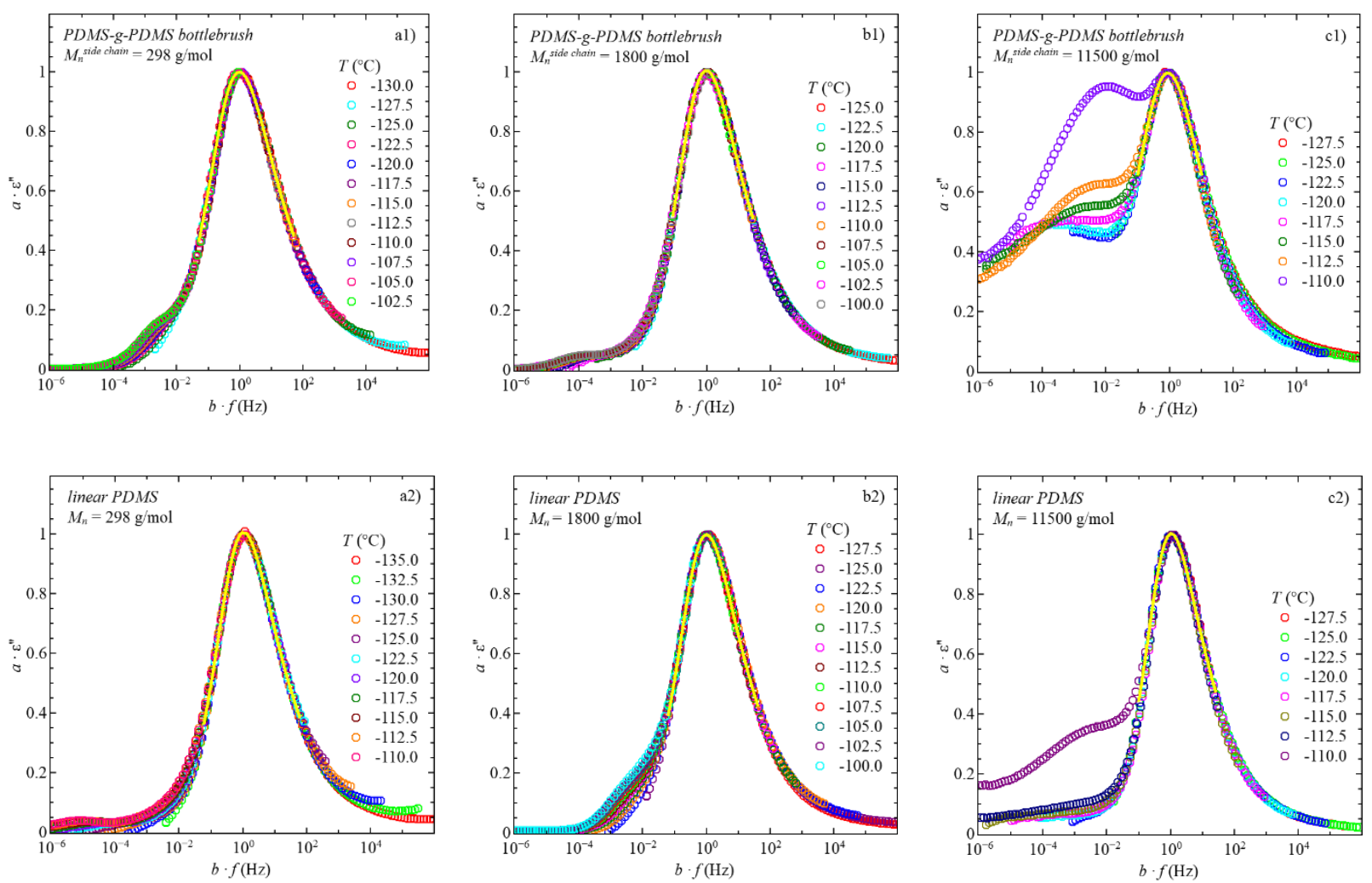

Figure 5. Normalized imaginary part of the permittivity, $a \cdot \epsilon^{\prime \prime}$, vs. normalized frequency, $b \cdot f$, for the PDMS-g-PDMS bottlebrush polymers (top) compared with the respective linear PDMS side chains (bottom). Top row: a1: $M_{n}^{\text {side chain }}=298 \mathrm{~g} / \mathrm{mol}, \mathrm{b} 1: M_{n}^{\text {side chain }}=1800 \mathrm{~g} / \mathrm{mol}$, c1: $M_{n}^{\text {side chain }}=11500 \mathrm{~g} / \mathrm{mol}$. Bottom row: a2: $M_{n}=298 \mathrm{~g} / \mathrm{mol}, \mathrm{b} 2: M_{n}=1800 \mathrm{~g} / \mathrm{mol}$, c2: $M_{n}=11500 \mathrm{~g} / \mathrm{mol}$. Solid lines represent the best descriptions with the Havriliak-Negami function.

Differences arising from the onset of the crystallization are visible at the low frequency sides.

263 In case of the short and middle side chain length, this little onset is not developed enough to perform

264 an adequate analysis for the $\alpha_{c}$-relaxation. In contrast, for the bottlebrush polymer and the single

265 side chains with $M_{n}^{\text {side chain }}=11500 \mathrm{~g} / \mathrm{mol}$ (Figure $5 \mathrm{c} 1$ and $\mathrm{c} 2$ ) pronounced deviations, caused

266 by the $\alpha_{c}$-relaxation, are visible at the left side.

The temperature independent shape of the $\alpha$-relaxation peak simplifies the description of the

268 data sets and allows to describe all temperatures by the HN model function. The parameters $\beta$ and

$269 \gamma$ are hereby shared over all temperatures. ${ }^{11,37}$ The associated fit values of the relaxation peak can 
270 be found in the Supporting Information. Therefore, the relaxation time associated with each single

271 temperature can be calculated from the shift factors used for the normalization. This leads to the

272 Havriliak-Negami relaxation time, $\tau_{H N}$, which is transformed to the relaxation time, $\tau_{s}$, connected

273 to the position of the relaxation peak, by using equation (10).

274 Figure 6 illustrates the relaxation time dependence on the reciprocal temperature, which is a

275 more convenient way to compare the relaxation behavior of the different materials. Hereby the

276 temperature dependence follows the empirical VFT-law (equation (11)). The resulting fit parame-

277 ters can be found in Table 3.

278 All samples have in common that the relaxation time decreases continuously with increasing 279 temperature. However, comparing the bottlebrush polymer with the respective side chains shows 280 an increased relaxation time. This effect depends on the molecular weight of the side chains and

281 disappears for sufficiently high $M_{n}^{\text {side chain }}$. For the shortest side chain length (Figure 6a) the re282 laxation times are slowed down by $\sim 2.5$ orders of magnitude and those for the middle side chains 283 by $\sim 1.5$ orders of magnitude (Figure 6 b). Upon increasing the side chain length further, to $284 \quad M_{n}^{\text {side }}$ chain $=11500 \mathrm{~g} / \mathrm{mol}$, both samples, bottlebrush and single side chain, coincide over the en285 tire temperature range and no increase of the relaxation time is observable (Figure 6c). 

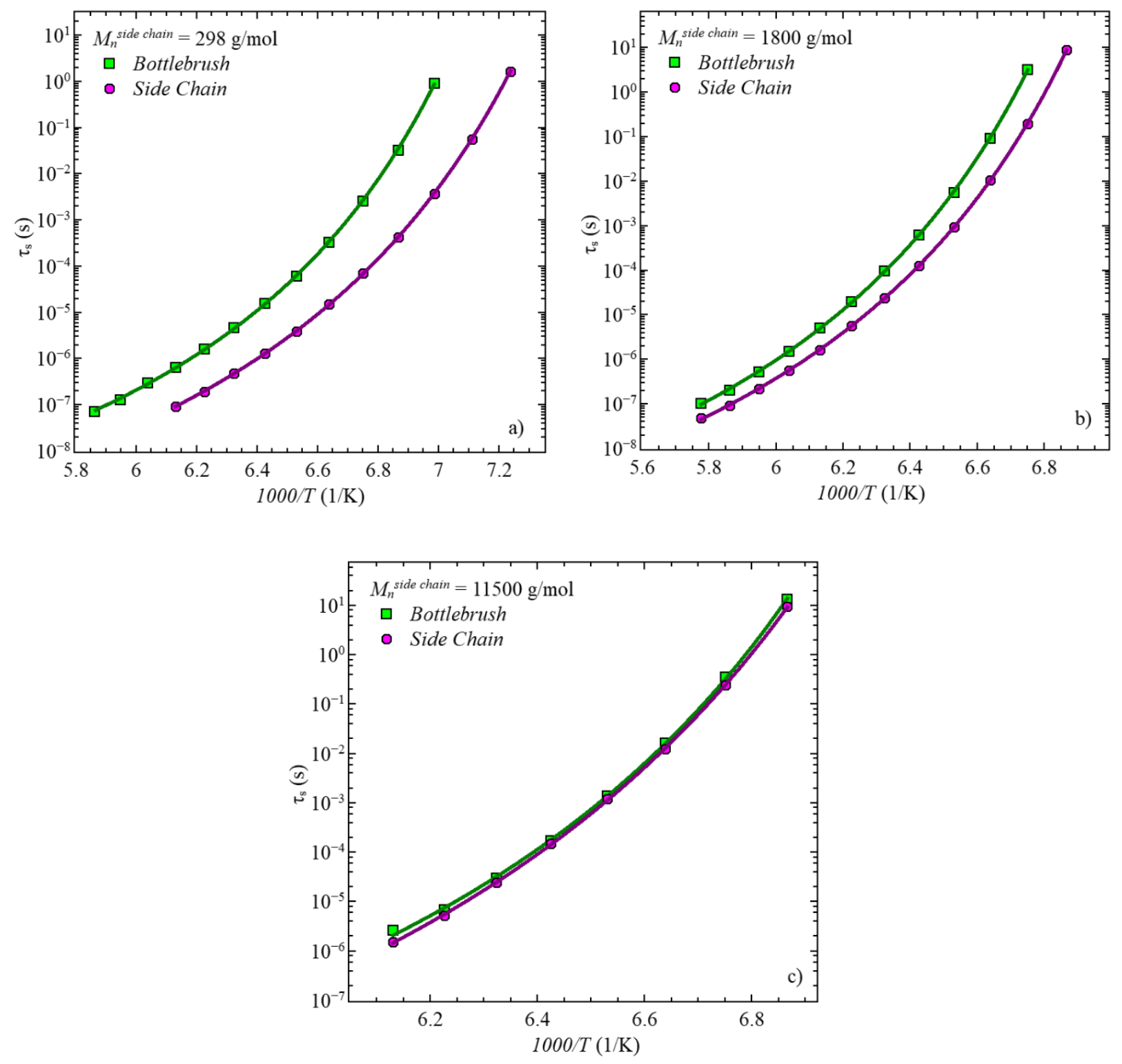

Figure 6. Segmental relaxation time, $\tau_{s}$, vs. 1000/T for the PDMS-g-PDMS bottlebrush polymer compared with their respective single linear PDMS side chains. a) $M_{n}^{\text {side chain }}=298 \mathrm{~g} / \mathrm{mol}$, b) $M_{n}^{\text {side chain }}=1800 \mathrm{~g} / \mathrm{mol}$, c) $M_{n}^{\text {side chain }}=11500 \mathrm{~g} / \mathrm{mol}$. Solid lines are the best description with the VFT equation. 
Table 3. Fit parameter for describing the temperature dependence of the segmental relaxation times from PDMS-g-PDMS bottlebrush polymer and their respective single side chain.

\begin{tabular}{c|c|c|c|c}
\hline \multirow{3}{*}{ Bottlebrush } & Sample & $\tau_{\infty}(\mathrm{s})$ & $A(\mathrm{~K})$ & $T_{0}(\mathrm{~K})$ \\
\cline { 2 - 5 } & Short & $(12.3 \pm 2.3) \cdot 10^{-13}$ & $508 \pm 11$ & $124.5 \pm 0.3$ \\
\cline { 2 - 5 } & Middle & $(13.4 \pm 3.9) \cdot 10^{-14}$ & $602 \pm 17$ & $128.6 \pm 0.4$ \\
\cline { 2 - 5 } & Long & $(4.6 \pm 2.3) \cdot 10^{-14}$ & $654 \pm 23$ & $126.0 \pm 0.4$ \\
\hline \multirow{3}{*}{ Side Chain } & Short & $(23.1 \pm 1.8) \cdot 10^{-14}$ & $572 \pm 4$ & $118.8 \pm 0.1$ \\
\cline { 2 - 5 } & Middle & $(92.3 \pm 6.1) \cdot 10^{-15}$ & $608 \pm 4$ & $126.7 \pm 0.1$ \\
\cline { 2 - 5 } & Long & $(5.6 \pm 1.3) \cdot 10^{-15}$ & $759 \pm 12$ & $123.9 \pm 0.2$ \\
\hline
\end{tabular}

For each graph, the relaxation time of chains covalently bonded to a backbone is compared to

of the lowest molar mass, equivalent to only four repeating units. Increasing the number of repeat-

298 ing units leads to a reduction of the differences in relaxation times, resulting in nearly overlapping

299 VFT-graphs for the longest side chains. This is a strong indication that those segments in close

300 proximity to the junction are affected the strongest, while there is less influence farther away from

301 the backbone. This suggests a critical length above which segments have the same relaxation time

302 as free chains. It may also suggest a gradual change from the inner to the outermost segments, that

303 may even be considered as a gradient of relaxation times from strongly confined, at the junction,

304 to free relaxation, equivalent to a linear chain.

305 Segmental relaxation is always connected to the glass transition temperature, which can be

306 defined as the temperature, at which the condition for the relaxation time $\tau_{s}=100 \mathrm{~s}$, i.e., $T_{g}=$

$307 T\left(\tau_{s}=100 \mathrm{~s}\right)$ is fulfilled. ${ }^{22}$ Extracted values for the single side chains, as well as for the respective

308 bottlebrush polymers are summarized in Table 4. Here differences are visible, depending on the 
309 architecture and the molecular weight. In general, the glass transition temperature of the bottlebrush

310 polymers is above the respective single side chains but in the same region. This trend matches the

311 differences in segmental relaxation times. Similar results were also found for different hetero- and

312 homopolymer bottlebrushes. ${ }^{13,38-39}$

Table 4. Extracted glass transition temperature, $T_{g}$, from dielectric spectroscopy measurement for the single linear PDMS side chains and the respective bottlebrush polymers.

\begin{tabular}{c|c|c|c}
\hline & Sample & $T_{g}\left({ }^{\circ} \mathrm{C}\right)$ & $T_{g}(K)$ \\
\hline \multirow{3}{*}{ Bottlebrush } & Short & $-132.8 \pm 0.2$ & $140.4 \pm 0.2$ \\
\cline { 2 - 4 } & Middle & $-127.0 \pm 0.5$ & $146.2 \pm 0.5$ \\
\cline { 2 - 4 } & Long & $-128.6 \pm 0.7$ & $144.6 \pm 0.7$ \\
\hline \multirow{3}{*}{ Side Chains } & Short & $-137.3 \pm 0.1$ & $135.8 \pm 0.1$ \\
\cline { 2 - 4 } & Middle & $-128.8 \pm 0.1$ & $144.3 \pm 0.1$ \\
\cline { 2 - 4 } & Long & $-128.9 \pm 0.2$ & $144.3 \pm 0.2$ \\
\hline
\end{tabular}

Therefore, the grafting process also increases the glass transition temperature, as expected by

316 the slowed down segmental relaxation time. However, the temperature dependence of the relaxa-

317 tion times is still the same, independent of the architecture, which is in contrast to heteropolymer

318 bottlebrush polymers. ${ }^{13}$

\section{Conclusion}

321 We have investigated the segmental relaxation behavior of PDMS-g-PDMS bottlebrush poly-

322 mers depending on the side chain lengths. Therefore, three bottlebrushes with different side chain

323 molecular weights have been synthesized, whereby in all cases the backbone length was kept 
324 constant. This results in increasing cross-sectional radii, investigated by small-angle neutron scat-

325 tering. The connected dynamical changes, inherent with the different side chain lengths, have been

326 examined by broadband dielectric spectroscopy, measuring the segmental relaxation times, $\tau_{s}$. By

327 comparing the segmental relaxation behavior of the bottlebrush polymers with the respective single

328 side chains, an increase in the segmental relaxation times is visible. Hereby, the strongest effect is

329 apparent in the sample with the shortest side chains. By increasing the side chain length, the effect

330 of an increased $\tau_{s}$ decreases. Finally, for the sample with the longest side chains, the segmental

331 relaxation times coincide over the whole temperature range. This effect of the increased relaxation

332 times originates from the grafting process and is independent of the grafting density. Consequently,

333 the glass transition temperatures of bottlebrush polymers can be approximated with equal or higher

334 values compared to those for the linear polymers equivalent to the side chains.

335

336 Acknowledgements

337 We acknowledge funding by the U.S. Department of Energy (DoE) under grant DE-SC0019050.

338 We are also grateful for the access to the neutron scattering instruments, provided by Australian

339 Nuclear Science and Technology Organisation (ANSTO).

\section{Supporting Information}

342 The supporting information is available free of charge at http://pubs.acs.org, including the experi-

343 mental procedures, additional GPC-chromatograms, ${ }^{1} \mathrm{H}$ NMR, additional SANS fit parameter, fit

344 values of the dielectric spectra, and temperature dependence of the relaxation times of $\alpha$ - and $\alpha_{c^{-}}$

345 relaxation for the long chain sample. 


\section{References}

$348 \quad$ 1. $\quad$ Polymeropoulos, G.; Zapsas, G.; Ntetsikas, K.; Bilalis, P.; Gnanou, Y.; Hadjichristidis, N., 349 50th Anniversary Perspective: Polymers with Complex Architectures. Macromolecules 2017, 50 350 (4), 1253-1290.

$351 \quad 2 . \quad$ Pesek, S. L.; Xiang, Q.; Hammouda, B.; Verduzco, R., Small-Angle Neutron Scattering 352 Analysis of Bottlebrush Backbone and Side Chain Flexibility. Journal of Polymer Science Part B:

353 Polymer Physics 2017, 55 (1), 104-111.

354 3. Pesek, S. L.; Li, X.; Hammouda, B.; Hong, K.; Verduzco, R., Small-Angle Neutron 355 Scattering Analysis of Bottlebrush Polymers Prepared via Grafting-Through Polymerization. 356 Macromolecules 2013, 46 (17), 6998-7005.

357 4. Rathgeber, S.; Pakula, T.; Wilk, A.; Matyjaszewski, K.; Beers, K. L., On the Shape of Bottle-Brush Macromolecules: Systematic Variation of Architectural Parameters. J Chem Phys 2005, 122 (12), 124904.

360 5. Daniel, W. F.; Burdynska, J.; Vatankhah-Varnoosfaderani, M.; Matyjaszewski, K.; Paturej, J.; Rubinstein, M.; Dobrynin, A. V.; Sheiko, S. S., Solvent-Free, Supersoft and Superelastic Bottlebrush Melts and Networks. Nat Mater 2016, 15 (2), 183-9.

363 6. $\quad$ Pesek, S. L.; Lin, Y.-H.; Mah, H. Z.; Kasper, W.; Chen, B.; Rohde, B. J.; Robertson, M. L.; 364 Stein, G. E.; Verduzco, R., Synthesis of Bottlebrush Copolymers based on Poly(dimethylsiloxane) 365 for Surface Active Additives. Polymer 2016, 98, 495-504.

366 7. Müllner, M., Molecular Polymer Brushes in Nanomedicine. Macromolecular Chemistry 367 and Physics 2016, 217 (20), 2209-2222.

368 8. Dalsin, S. J.; Hillmyer, M. A.; Bates, F. S., Linear Rheology of Polyolefin-Based 369 Bottlebrush Polymers. Macromolecules 2015, 48 (13), 4680-4691.

$3709 . \quad$ Dalsin, S. J.; Hillmyer, M. A.; Bates, F. S., Molecular Weight Dependence of Zero-Shear 371 Viscosity in Atactic Polypropylene Bottlebrush Polymers. ACS Macro Letters 2014, 3 (5), 423372427.

373 10. Mauro, J. C.; Yue, Y.; Ellison, A. J.; Gupta, P. K.; Allan, D. C., Viscosity of Glass-Forming 374 Liquids. Proceedings of the National Academy of Sciences 2009, 106 (47), 19780. 
11. Hintermeyer, J.; Herrmann, A.; Kahlau, R.; Goiceanu, C.; Rössler, E. A., Molecular Weight Dependence of Glassy Dynamics in Linear Polymers Revisited. Macromolecules 2008, 41 (23), 9335-9344.

12. Staropoli, M.; Raba, A.; Hövelmann, C. H.; Appavou, M.-S.; Allgaier, J.; Krutyeva, M.; Pyckhout-Hintzen, W.; Wischnewski, A.; Richter, D., Melt Dynamics of Supramolecular Comb Polymers: Viscoelastic and Dielectric response. Journal of rheology 2017, 61 (6), 1185-1196.

13. Grigoriadis, C.; Nese, A.; Matyjaszewski, K.; Pakula, T.; Butt, H.-J.; Floudas, G., Dynamic Homogeneity by Architectural Design - Bottlebrush Polymers. Macromolecular Chemistry and Physics 2012, 213 (13), 1311-1320.

14. Nikovia, C.; Theodoridis, L.; Alexandris, S.; Bilalis, P.; Hadjichristidis, N.; Floudas, G.; Pitsikalis, M., Macromolecular Brushes by Combination of Ring-Opening and Ring-Opening Metathesis Polymerization. Synthesis, Self-Assembly, Thermodynamics, and Dynamics. Macromolecules 2018, 51 (21), 8940-8955.

15. Beiner, M.; Huth, H., Nanophase Separation and Hindered Glass Transition in Side-Chain Polymers. Nature Materials 2003, 2 (9), 595-599.

16. Gerstl, C.; Schneider, G. J.; Fuxman, A.; Zamponi, M.; Frick, B.; Seydel, T.; Koza, M.; Genix, A. C.; Allgaier, J.; Richter, D.; Colmenero, J.; Arbe, A., Quasielastic Neutron Scattering Study on the Dynamics of Poly(alkylene oxide)s. Macromolecules 2012, 45 (10), 4394-4405.

17. Pedersen, J. S.; Schurtenberger, P., Cross-Section Structure of Cylindrical and PolymerLike Micelles from Small-Angle Scattering Data. I. Test of Analysis Methods. Journal of Applied Crystallography 1996, 29 (6), 646-661.

18. Chen, W.-R.; Butler, P. D.; Magid, L. J., Incorporating Intermicellar Interactions in the Fitting of SANS Data from Cationic Wormlike Micelles. Langmuir 2006, 22 (15), 6539-6548.

19. Daoud, M.; Cotton, J. P., Star Shaped Polymers : A Model for the Conformation and its Concentration Dependence. J. Phys. France 1982, 43 (3), 531-538.

20. Pedersen, J. S.; Schurtenberger, P., Scattering Functions of Semiflexible Polymers with and without Excluded Volume Effects. Macromolecules 1996, 29 (23), 7602-7612.

21. Dozier, W. D.; Huang, J. S.; Fetters, L. J., Colloidal Nature of Star Polymer Dilute and Semidilute Solutions. Macromolecules 1991, 24 (10), 2810-2814. 
22. Kremer, F.; Schönhals, A., Broadband Dielectric Spectroscopy. Springer Berlin Heidelberg: 2012.

406 23. Strobl, G. R., The Physics of Polymers: Concepts for Understanding Their Structures and

Behavior. Springer Berlin Heidelberg: 2013.

24. Díaz-Calleja, R., Comment on the Maximum in the Loss Permittivity for the

Havriliak-Negami Equation. Macromolecules 2000, 33 (24), 8924-8924.

25. Boersma, A.; Turnhout, J.; Wübbenhorst, M., Dielectric Characterization of a Thermotropic Liquid Crystalline Copolyesteramide: 1. Relaxation Peak Assignment. Macromolecules 1998, 31, 7453-7460.

26. Gerstl, C.; Schneider, G. J.; Pyckhout-Hintzen, W.; Allgaier, J.; Richter, D.; Alegría, A.; Colmenero, J., Segmental and Normal Mode Relaxation of Poly(alkylene oxide)s Studied by

Dielectric Spectroscopy and Rheology. Macromolecules 2010, 43 (11), 4968-4977. Bonding on Dynamics of Hydroxyl-Terminated Polydimethylsiloxane. Macromolecules 2016, 49 (8), 3138-3147.

28. Sokolova, A.; Christoforidis, J.; Eltobaji, A.; Barnes, J.; Darmann, F.; Whitten, A. E.; de

29. Sokolova, A.; Whitten, A. E.; de Campo, L.; Christoforidis, J.; Eltobaji, A.; Barnes, J.; Darmann, F.; Berry, A., Performance and Characteristics of the BILBY Time-of-Flight SmallAngle Neutron Scattering Instrument. Journal of Applied Crystallography 2019, 52 (1), 1-12.

30. Arnold, O.; Bilheux, J. C.; Borreguero, J. M.; Buts, A.; Campbell, S. I.; Chapon, L.; Doucet, M.; Draper, N.; Ferraz Leal, R.; Gigg, M. A.; Lynch, V. E.; Markvardsen, A.; Mikkelson, D. J.; Mikkelson, R. L.; Miller, R.; Palmen, K.; Parker, P.; Passos, G.; Perring, T. G.; Peterson, P. F.; Ren, S.; Reuter, M. A.; Savici, A. T.; Taylor, J. W.; Taylor, R. J.; Tolchenov, R.; Zhou, W.; Zikovsky, J., Mantid - Data Analysis and Visualization Package for Neutron Scattering and $\mu$ SR Experiments. Nuclear Instruments and Methods in Physics Research Section A: Accelerators, Spectrometers, Detectors and Associated Equipment 2014, 764, 156-166. Branched Polysiloxanes with Controlled Branching and Functionalization by Anionic RingOpening Polymerization. Macromolecules 2003, 36 (11), 3890-3897. 
435 32. Bichler, K. J.; Jakobi, B.; Huber, S. O.; Gilbert, E. P.; Schneider, G. J., Structural Analysis of Ultrasoft PDMS-g-PDMS Shell-Only Particles. Macromolecules 2020, 53 (1), 78-89.

437 33. Pongkittiphan, V.; Theodorakis, E. A.; Chavasiri, W., Hexachloroethane: a Highly Efficient 438 Reagent for the Synthesis of Chlorosilanes from Hydrosilanes. Tetrahedron letters 2009, 50 (36), 439 5080-5082.

$440 \quad 34 . \quad$ van Genabeek, B.; de Waal, B. F. M.; Gosens, M. M. J.; Pitet, L. M.; Palmans, A. R. A.; 441 Meijer, E. W., Synthesis and Self-Assembly of Discrete Dimethylsiloxane-Lactic Acid Diblock 442 Co-oligomers: The Dononacontamer and Its Shorter Homologues. Journal of the American 443 Chemical Society 2016, 138 (12), 4210-4218.

$444 \quad 35 . \quad$ Rubinstein, M.; Colby, R. H., Polymer Physics. OUP Oxford: 2003.

445 36. Lund, R.; Alegria, A.; Goitandia, L.; Colmenero, J.; González, M. A.; Lindner, P., 446 Dynamical and Structural Aspects of the Cold Crystallization of Poly (Dimethylsiloxane)(PDMS). 447 Macromolecules 2008, 41 (4), 1364-1376.

448 37. Lunkenheimer, P.; Loidl, A., Dielectric Spectroscopy of Glass-Forming Materials: $\alpha-$ 449 Relaxation and Excess Wing. Chemical Physics 2002, 284 (1), 205-219.

$450 \quad 38 . \quad$ Tsukahara, Y.; Namba, S.-i.; Iwasa, J.; Nakano, Y.; Kaeriyama, K.; Takahashi, M., Bulk 451 Properties of Poly(macromonomer)s of Increased Backbone and Branch Lengths. Macromolecules 452 2001, 34 (8), 2624-2629. Response of a Series of Densely Branched Brush Polymers. Macromolecules 2011, 44 (17), 69356943. 